\title{
ERRATUM
}

\section{ASPP and cancer}

Giuseppe Trigiante and Xin Lu

Nature Rev. Cancer 6, 217-226 (2006)

The correct title of this article should be ASPP and cancer, not ASPPs and cancer as originally published. This change will enable the article to be found using the standard search term ASPP in the Entrez-PubMed database. 